\title{
Key roles of CCCTC-binding factor in cancer evolution and development
}

\author{
Zishuai $\mathrm{Li}^{\dagger}$, Xinyu Zhou ${ }^{\dagger}$, Shiliang Cai ${ }^{\dagger}$ Junyan Fan, Zhimin Wei, Yifan Chen, Guangwen Cao* \\ Department of Epidemiology, Faculty of Naval Medicine, Second Military Medical University, Shanghai 200433, China
}

${ }^{\dagger}$ These authors share the first authorship.

*Correspondence: Guangwen Cao, Department of Epidemiology, Faculty of Naval Medicine, Second Military Medical University, 8 Panshan Road, Shanghai 200433, China. gcao@smmu.edu.cn

Academic Editor: Derek M. Dykxhoorn, University of Miami Miller School of Medicine, USA

Received: August 19, 2021 Accepted: November 20, 2021 Published: December 31, 2021

Cite this article: Li Z, Zhou X, Cai S, Fan J, Wei Z, Chen Y, et al. Key roles of CCCTC-binding factor in cancer evolution and development. Explor Med. 2021;2:516-26. https://doi.org/10.37349/emed.2021.00068

\begin{abstract}
The processes of cancer and embryonic development have a partially overlapping effect. Several transcription factor families, which are highly conserved in the evolutionary history of biology, play a key role in the development of cancer and are often responsible for the pivotal developmental processes such as cell survival, expansion, senescence, and differentiation. As an evolutionary conserved and ubiquitously expression protein, CCCTC-binding factor (CTCF) has diverse regulatory functions, including gene regulation, imprinting, insulation, $\mathrm{X}$ chromosome inactivation, and the establishment of three-dimensional (3D) chromatin structure during human embryogenesis. In various cancers, CTCF is considered as a tumor suppressor gene and plays homeostatic roles in maintaining genome function and integrity. However, the mechanisms of CTCF in tumor development have not been fully elucidated. Here, this review will focus on the key roles of CTCF in cancer evolution and development (Cancer Evo-Dev) and embryogenesis.
\end{abstract}

\section{Keywords}

CCCTC-binding factor, cancer evolution and development, embryogenesis

\section{Introduction}

CCCTC-binding factor (CTCF) is a multivalent 11-zinc finger (ZF) protein that binds tens of thousands of sites in the human genome [1]. Initially identified as transcriptional regulator of chicken MYC proto-oncogene (c-myc gene) in 1990 [2, 3], CTCF was later found to mediate gene imprinting at the H19 imprinted maternally expressed transcript/insulin-like growth factor 2 (H19/Igf2) locus [4]. Follow-up studies indicated that CTCF interferes with the interaction between enhancers and promoters because of its binding sites frequent presence in various chromosomal structural boundaries, such as the topologically associated domains (TADs) $[5,6]$. Increasing evidence indicated that CTCF protein is highly conserved from Drosophila to humans [7]. CTCF is one of the key regulatory proteins in vertebrates, and its knockout leads high in utero embryonic lethality [8]. CTCF can participate in a variety of biological processes and have different functions, including insulator function [9], regulation 
of gene transcription [10], gene imprinting (Figure 1) [11], X chromosome inactivation [12, 13], affecting messenger RNA (mRNA) alternative splicing as well as nucleosome rearrangement and DNA replication $[14,15]$. Recently, high-throughput chromosome conformation capture (Hi-C) indicated that CTCF has dynamic roles as regulator of imprinted loci and governing higher-order chromatin architecture other than enhancer blocking activity $[5,16]$. The latest evidences revealed that CTCF is directly involved in the transcriptional modulation of various key factors of cellular cycle control, apoptosis, senescence, and differentiation $[17,18]$. Indeed, ectopic expression of CTCF in several human tumoral cell lines inhabits cell division and clonogenicity [18]. Somatic mutations at CTCF-binding sites, which can abrogate the CTCF-mediated spatial folding of chromosomes [19], have been profoundly found in various human cancers [20]. Interestingly, CTCF also has a key role in the establishment of three-dimensional (3D) chromatin structure during human embryogenesis [21]. The basic framework of the hypothesis of cancer evolution and development (Cancer Evo-Dev) is shown in Figure 2. The complex interactions between host genetic susceptibility and environmental exposure [such as hepatitis B virus (HBV) infections] induced immune system disorder. The dysfunctional immune system activates and maintains chronic inflammation with the permanent existence of HBV in host. Under the inflammatory microenvironment, the imbalance of mutagenic factors and mutant repair factors, cooperating with HBV integration and epigenetic modulations, promotes HBV-related somatic mutations and HBV mutations. A majority of hepatocytes with genomic mutations and HBV variants are eliminated in the survival competition in inflammatory microenvironments. Only a small part of cells survive by alternating survival signaling pathways and exhibit "stemness" characteristics. The survivals gradually evolved to be cancer stem cells, which drives the development and evolution of cancer [22]. According to the Cancer Evo-Dev hypothesis, CTCF is considered as an essential regulator during cancer cells "backward evolution" and "retro-differentiation".

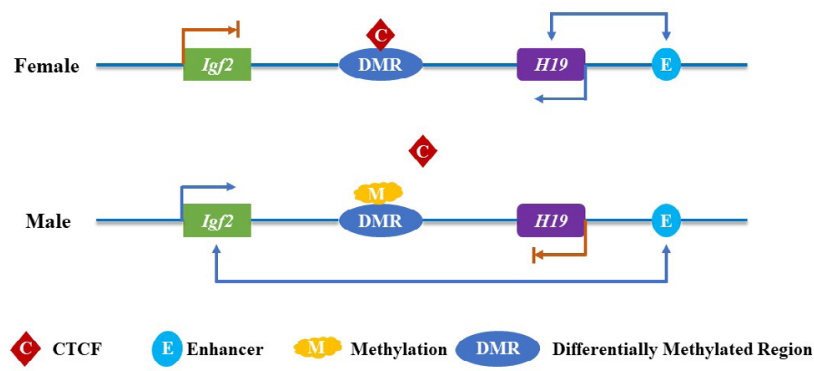

Figure 1. Gene imprinting. The methylation of the paternal allele DMR affects the binding of CTCF, and the enhancer downstream of $\mathrm{H} 19$ can interact with the Igf2 over a long distance to promote its expression. On the contrary, the maternal allele DMR is not methylated, which means CTCF can bind to it and then block the interaction between the downstream enhancers of $\mathrm{H} 19$ and the promoters of Igf2. As a result, the expression of lgf2 is turned off, but H19 can still be expressed under the action of downstream enhancers

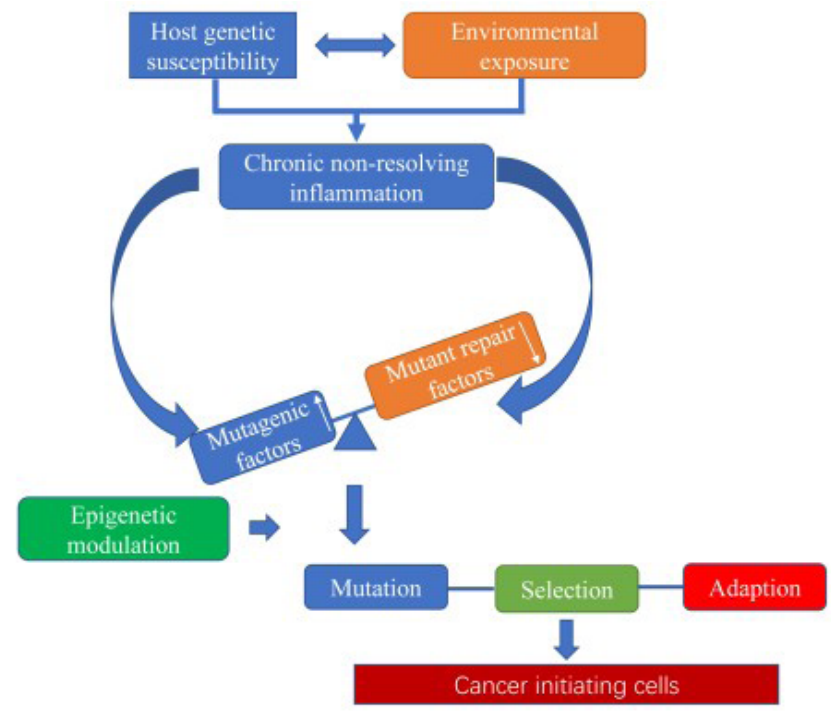

Figure 2. Basic framework of Cancer Evo-Dev 


\section{CTCF is essential to establish 3D chromatin structure during human embryogenesis}

There is a growing consensus that a hierarchical chromatin structure is established within the nucleus in the interphase of the cell cycle [23, 24]. The spatial folding of chromosomes and their organization, which is largely mediated by CTCF, have profound effects on gene expression [25, 26]. Genetically inducible CTCF deletion in several specific cell types, including oocytes, lymphocytes, and cardiomyocytes, leads to organ-specific failure [27, 28]. Knocking out the CTCF of the oocyte seriously disrupts the entry of the fertilized oocyte into the blastocyst stage [28]. Furthermore, homozygous deletion of CTCF at the whole embryo level results in embryo death [8], which is characterized by aberrant enhancer-promoter interactions and transcriptional dysregulation [29]. However, albeit numerous studies have explored the function of CTCF binding, the in vivo roles of CTCF during embryonic development are largely unknown.

Recent studies revealed that TAD structures are totally lost in human 2-cell embryos, are at a low level in 8-cell embryos, and then gradually established during embryonic development [21]. As an essential hierarchical structural feature of chromatin organization, A/B compartmentalization is absent in human 2-cell embryos and reformed during embryogenesis [21, 30, 31]. Unlike in mature mouse sperm, TAD structures are absent in human sperm. Previous studies have shown that depletion of CTCF can lead to the disruption of TADs, suggesting that the lack of CTCF may contribute to the loss of TAD structures in human sperm [21,32]. Immature TAD boundaries gained at the 2-cell embryos tend to locate around housekeeping genes, which may promote the expression levels of housekeeping genes. In addition, both CTCF expression and TAD establishment in human embryos require human zygotic genome activation (ZGA). Further studies suggested that CTCF expression is required, but is not the only factor needed, for TAD establishment during human ZGA [21].

\section{The regulatory role of CTCF in tumors}

CTCF can induce both cell cycle progression or arrest to accomplish its function [17], which is dependent by cell-specific CTCF-binding DNA sequences (CTSs), protein partners and chromatin long-range interactions [33]. CTCF can repress cell growth and colony formation suggesting a suppressor role of CTCF [34, 35].

\section{CTCF/CTCF like maintains genome stability}

It is reported that CTCF depletion activates DNA damage response and increases the risk of chromosomal instability [36]. DNA damage signaling, Mre11 (MRE11 homolog, double strand break repair nuclease)-Rad50 (RAD50 double strand break repair protein)-Nbs1 (nijmegen breakage syndrome 1) complex and CTCF DNA-binding domain promote CTCF enrichment at DNA damage sites [36]. As the core DNA-binding protein of homologous recombination (HR), RAD51 is overexpressed in a variety of tumors [37]. CTCF participates in HR repair of DNA double strand breaks by interacting with RAD51 and promoting the formation of RAD51 repair foci [38]. CTCFL (CTCF like), otherwise known as Brother of the Regulator of Imprinted Sites (BORIS), is a male system-specific protein with the same 11-zinc finger structure as CTCF [38]. In non-small cell lung cancer (NSCLC), BORIS suppresses DNA damage and promotes cisplatin resistance by enhancing the mismatch repair system of cancer cells [39].

\section{CTCF, epigenetic regulation and cancer}

Gene methylation detection showed that DNA methylation at the CpG sites in the CTCF coding gene caused the CTCF binding sites to be blocked [40]. The function of CTCF in regulating gene imprinting suggested that the binding of CTCF to the target gene is methylation sensitive [4]. The aberrant DNA methylation of key regulate regions can hinder CTCF binding, which may lead to epigenetic silencing of tumor suppressor loci or lead to activation of oncogenes [41]. Large tumor suppressor (LATS) kinase can be activated by a variety of stress responses, such as glucose deficiency, and then phosphorylates 
downstream pathways to promote cell survival, while abnormal LATS activation promotes tumorigenesis. LATS kinase directly phosphorylates CTCF at the ZF junction, inhibiting its DNA binding activity [42]. Recent studies indicated that DNA methylation-regulated alternative cleavage and polyadenylation (APA) requires CTCF and the cohesin complex [43]. Epigenetic inactivation of Ras association domain family 1 isoform A (RASSF1A) and E-cadherin (CDH1) in breast cancer is associated with alternations in CTCF recognition sites [44]. Aberrant DNA methylation can inhibit CTCF-mediated silencing of BCL6 transcription repressor (BCL6) gene, thus increasing the expression of proto-oncogene BCL6 in lymphoma [45]. In addition, alternations in $C T C F$ gene in endometrial cancer can promote tumorigenesis by promoting cell survival and changing cell polarity [46].

CTCF hemizygous deletions are frequently found in various human tumors [47]. Loss of a single CTCF allele $\left(\mathrm{Ctcf}^{+/}\right)$significantly increases the risk of cancer and enhances malignant progression [40]. CTCF hemizygosity dysregulates cancer-related pathways, such as Ras, Ras-mitogen-activated protein kinase (Ras-MAPK) and extracellular signal-regulated kinase 1/2 (ERK1/2) signaling pathways [48]. Ctcft/ mice remain a disorder methylation status, and even lead to a modest overall increase levels of genome-wide DNA methylation in lung [40]. Chromosome 16q22.1 is a common deletion region in various epithelial cancers, and CTCF is located on this region. In prostate cancer cells, knockdown of CTCF results in hypermethylation of the CTCF/cohesin-binding sites (CBSs). Prostate and breast cancers with insufficient CTCF copy number show increased DNA hypermethylation events in vivo [49]. In addition, high frequency mutations of CBSs also lead to disorders of specific sites hypermethylation and promote the occurrence and development of cancer [43].

\section{CTCF can modulate key tumor-related genes}

CTCF has been shown to modulate the expression of various cancer-associated genes. c-myc plays vital roles in tumorigenesis, embryogenesis, and somatic cells reprogramming [50-52]. CTCF exerts divergent roles on $c-m y c$ regulation by binding various sequences at $c$-myc promoter [1]. In myeloid cells, overexpression of CTCF decreases $c$-myc levels and growth rate, which promotes myeloid differentiation and induces cell cycle arrest in numerous tumoral cells $[34,53]$. In most malignant tumors, telomerase is activated to prevent telomere shortening, which confers high proliferative capacity to tumoral cells $[54,55]$. As one of the telomerase components, human telomerase reverse transcriptase (hTERT) is only expressed in telomerase-positive cells, and depletion of CTCF induced hTERT transcription in TERT-negative cells $[56,57]$. However, CTCF is not able to exert a transcriptional repressive function because of DNA-methylation or BORIS-binding in TERT-positive cells [58]. Moreover, in cancer cells, methylation of CTSs at hTERT exon inhibits CTCF-binding, which prevents hTERT repression [59]. Retinoblastoma $(R B)$ is considered a tumor suppressor gene and CTCF maintains $R B$ gene promoters at an active epigenetic status $[60,61]$. The inactivated of $R B$ gene family leads to the loss of cell cycle control and cancer. Other evidences showed that CTCF is also involved in an epigenetic balance at the cyclin-dependent kinase inhibitor 2A locus $(C D K N 2 A)$ and tumor protein p53 (TP53) gene promoters [58, 62]. In sum, by binding various CTSs, CTCF can modulate $c$-myc, $h T E R T, R B$, and other tumor-related genes, which emphasize the cell-type dependency of its tumor suppressor role.

\section{CTCF/Cohesin complex is associated with cancer development}

Chromosome architecture has different levels: euchromatin (A) and heterochromatin (B) compartments are corresponding to megabase-scale, TADs are corresponding to sub-megabase scale and smaller loop structures correspond to tens of kilobases level [63]. As a sister chromatid cohesin molecule, cohesin was also found to modulate higher-order chromosome architecture by cooperating with CTCF. CTCF interacts with the cohesin through two different domains at the N-terminal. Amino acid 222-231 binds to the cohesin's conserved essential surface, which is a composite interface formed by stromal antigen (SA) and RAD21 cohesin complex component (RAD21) subunits. This motif is known as YxF motif in CTCF. Amino acid 23-27 binds to the cohesin protein PDS5A subunit. This motif is known as YxR motif in CTCF [64, 65]. Interestingly, CTCF assists cohesin to bind specific sites on chromosomes while its binding is independent of the presence of cohesin 
on chromatin [66]. Recent evidence indicated that cohesin catalyzes the folding of the genome into loops that are anchored by CTCF and CTCF enables chromatin loop formation by protecting cohesin against loop release [65]. CTCF/cohesin-mediated chromatin loops play an essential role in the maintenance of genome integrity [67]. Previous studies showed that mutations at CBSs and somatic mutations in the cohesin subunits were frequently detected in various cancers, while aberrant overexpression of the cohesin complex was also frequently found in several human malignancies [20,68,69]. Mutations in cohesin subunits and CBSs may promote genomic instability by perturbing proper long-range chromatin interactions. In cancer cells with chromosomal instability, CTCF/cohesin-mediated chromatin organization and DNA replication play an essential role in gene stable amplification [70]. As a member of cohesin family gene, stromal antigen 2 (STAG2) is one of the most commonly mutated genes in cancer. In Ewing's sarcoma, STAG2 disfunction is related to aggressive behavior. STAG2 loss of function orchestrates oncogenic transcription factors by changing CTCF-anchored loop extrusion [71]. RAD21 is an essential subunit of the cohesin complex. Interestingly, depletion of RAD21 in epithelial cancer cells induces epithelial to mesenchymal transition (EMT) while overexpression of RAD21 in mesenchymal cancer cells induces mesenchymal to epithelial transition (MET)specific expression patterns, suggesting that dynamic cohesin-mediated chromatin structures are responsible for the initiation and regulation of essential EMT-related cell fate changes in cancer [72].

\section{The function of BORIS/CTCF system in tumorigenesis}

CTCF is constitutively and widely expressed in normal tissues while BORIS protein exists normally only in the testis [73]. BORIS belongs to the cancer/testis gene family, which is only expressed in malignant tumors except male germ cells. The abnormal expression levels of BORIS RNA and protein, which are affected by DNA-methylations, related with the size of tumors and the degree of malignancy, otherwise the knockdown of BORIS induced apoptosis in tumorous cells [74]. CTCF and BORIS do not compete even they have the same recognition sites in normal somatic cells. However, expression of BORIS in BORIS-negative cells not only interferes with the normal functions such as growth inhibition of CTCF, but also leads to cell dysfunction, which leads to tumorigenesis due to the competitive binding of BORIS/CTCF gene family [75]. Recent studies indicated that ectopic expression of BORIS activates cancer testes antigens (CTA) and components of cancer relevant signaling pathways [76]. The test of sterile BORIS ${ }^{-/-} \mathrm{CTCF}^{+/-}$(compound mutant, CM) male mouses confirmed that combined depletion of BORIS/CTCF will lead to defection of meiotic recombination, increasing of apoptosis, and malformed spermatozoa [77]. Interestingly, BORIS/CTCF heterodimeric sites are enriched in both cancer and germ when the promotors and enhancers of cells are activated [78]. The low expression of spermatogenesis genes and aberrant expression of sterile genes in CM mouses indicated that joint action of BORIS/CTCF is essential for spermatogenesis program by restraining pre-meiotic genes and activating post-meiotic genes [77].

\section{CTCF and Cancer Evo-Dev}

Cancer development is characterized by an evolutionary process of "mutation-selection-adaptation", some highly conserved genes are highly expressed in the embryo, not expressed or low expressed in normal adult tissues, but highly expressed in cancer tissues, leading to reverse cell differentiation, malignant proliferation and enhanced migration capacity [22]. Recent evidences revealed that in normal prostate tissue, CTCF expression was negative to low, while in prostate cancers, CTCF expression was seen in 7,726 of 12,555 (61.5\%) tumors and was considered low in $44.6 \%$ and high in $17 \%$ of cancers [79]. CTCF expression is a feature of poor prognostic in prostate cancer, but CTCF is a dissatisfactory candidate biomarker because of its low predictive power [79]. Similarly, CTCF is frequently up-regulated in partial primary hepatocellular carcinoma (HCC) compared with non-neoplastic liver. Overexpression of CTCF is associated with shorter disease-free survival in patients and the absence of CTCF can lead to decreased motility and invasiveness of HCC cells [80]. In addition, chromosomal ring anchors bound by CTCF and cohesin are prone to continuous DNA breakage, and regions of translocation break points in various cancers are enriched in these anchors [67]. Continuous DNA break and repair provide opportunities for genetic mutations, and in the context of numerous mutations, cancer is constantly selecting and adapting. 
Furthermore, multiple cancer types accumulate CBS mutations and CBSs are major mutational hotspots in the noncoding cancer genome, which emphasize the significant role of CTCF in cancer evolution and development. In summary, CTCF hemizygotic mutations and CBSs mutations disrupt genomic stability and, in combination with epigenetic modifications such as methylation, promote the mutation and adaptive selection of cancer cells, thereby promoting the evolution and development of cancer.

\section{Conclusion}

CTCF, a highly conserved and multifunctional protein, contributes to formation of multi-dimensions genome and control of central signals to transcriptional networks [5]. CTCF plays an essential role in embryonic development and cancer development, but its molecular mechanism has not been fully elucidated. CTCF exerts a tumor suppressor role by regulating several key factors related to growth and development and regulating the higher-order structure of chromosomes, but its high expression in various cancer cells may promote the malignant proliferation and migration, and often predicts a poor prognosis [33, 79]. Although roles of CTCF in carcinogenesis have been intensively explored, more researches are needed to better understand the significant functions and mechanisms of CTCF in embryonic development and cancer development.

With the continuous development of modern bioinformatics and biotechnology, the combined use of a variety of high-throughput analysis techniques such as chromatin immunoprecipitation-chip (ChIP-chip), ChIP-qPCR, and so on can constantly find out the corresponding binding sites and regulatory models of CTCF with DNA, protein and RNA, which can further clarify the action mechanism of CTCF. The developed highly specific epigenome editing technology based on clustered regularly interspersed short palindromic repeats (CRISPR)/CRISPR-associated 9 (Cas9) as a broad prospect because it can modify the genome of specific regulatory elements [81]. For example, targeted editing of the DNA methylation status of CTCF binding sites can alter the expression of CTCF, thereby altering the expression of target genes by affecting the structure of advanced chromatin [82]. Therefore, the highly specific epigenome editing technology based on CRISPR/Cas9 may become an attractive epigenome-based cancer therapy in the next few years to regulate the occurrence and development of tumors.

\section{Abbreviations}

BORIS: Brother of the Regulator of Imprinted Sites

Cancer Evo-Dev: cancer evolution and development

CBSs: cohesin-binding sites

c-myc: chicken MYC proto-oncogene

CTCF: CCCTC-binding factor

CTSs: CCCTC-binding factor-binding DNA sequences

HBV: hepatitis B virus

hTERT: human telomerase reverse transcriptase

Igf2: insulin-like growth factor 2

LATS: large tumor suppressor

$R B$ : retinoblastoma

STAG2: stromal antigen 2

TADs: topologically associated domains

\section{Declarations}

\section{Author contributions}

$\mathrm{ZL}, \mathrm{XZ}$ and SC participated in the manuscript design, and writing. JF, ZW and YC contributed to the figures. GC extensively revised the manuscript. All authors contributed to manuscript revision, read and approved the submitted version. 


\section{Conflicts of interest}

The authors declare that they have no conflicts of interest.

Ethical approval

Not applicable.

\section{Consent to participate}

Not applicable.

Consent to publication

Not applicable.

\section{Availability of data and materials}

Not applicable.

\section{Funding}

This work was supported by grant 2015CB554006 from the National Key Basic Research Program of China (GC); grants 81673250, 91529305, and 81520108021 from the National Natural Science Foundation of China (GC). The funders had no role in study design, data collection and analysis, decision to publish, or preparation of the manuscript.

\section{Copyright}

(C) The Author(s) 2021.

\section{References}

1. Filippova GN, Fagerlie S, Klenova EM, Myers C, Dehner Y, Goodwin G, et al. An exceptionally conserved transcriptional repressor, CTCF, employs different combinations of zinc fingers to bind diverged promoter sequences of avian and mammalian c-myc oncogenes. Mol Cell Biol. 1996;16:2802-13.

2. Baniahmad A, Steiner C, Köhne AC, Renkawitz R. Modular structure of a chicken lysozyme silencer: involvement of an unusual thyroid hormone receptor binding site. Cell. 1990;61:505-14.

3. Lobanenkov VV, Nicolas RH, Adler VV, Paterson H, Klenova EM, Polotskaja AV, et al. A novel sequence-specific DNA binding protein which interacts with three regularly spaced direct repeats of the CCCTC-motif in the 5'-flanking sequence of the chicken c-myc gene. Oncogene. 1990;5:1743-53.

4. Hark AT, Schoenherr CJ, Katz DJ, Ingram RS, Levorse JM, Tilghman SM. CTCF mediates methylation-sensitive enhancer-blocking activity at the H19/Igf2 locus. Nature. 2000;405:486-9.

5. Vietri Rudan M, Barrington C, Henderson S, Ernst C, Odom DT, Tanay A, et al. Comparative Hi-C reveals that CTCF underlies evolution of chromosomal domain architecture. Cell Rep. 2015;10:1297-309.

6. Moore BL, Aitken S, Semple CA. Integrative modeling reveals the principles of multi-scale chromatin boundary formation in human nuclear organization. Genome Biol. 2015;16:110.

7. Ohlsson R, Lobanenkov V, Klenova E. Does CTCF mediate between nuclear organization and gene expression? Bioessays. 2010;32:37-50.

8. Fedoriw AM, Stein P, Svoboda P, Schultz RM, Bartolomei MS. Transgenic RNAi reveals essential function for CTCF in $H 19$ gene imprinting. Science. 2004;303:238-40.

9. Jia Z, Li J, Ge X, Wu Y, Guo Y, Wu Q. Tandem CTCF sites function as insulators to balance spatial chromatin contacts and topological enhancer-promoter selection. Genome Biol. 2020;21:75.

10. McGill BE, Barve RA, Maloney SE, Strickland A, Rensing N, Wang PL, et al. Abnormal microglia and enhanced inflammation-related gene transcription in mice with conditional deletion of Ctcf in Camk2a-Cre-expressing neurons. J Neurosci. 2018;38:200-19. 
11. Bell A, Felsenfeld G. Methylation of a CTCF-dependent boundary controls imprinted expression of the Igf2 gene. Nature. 2000;405:482-5.

12. Aeby E, Lee HG, Lee YW, Kriz A, Del Rosario BC, Oh HJ, et al. Decapping enzyme 1A breaks X-chromosome symmetry by controlling Tsix elongation and RNA turnover. Nat Cell Biol. 2020;22:1116-29.

13. Fang H, Bonora G, Lewandowski JP, Thakur J, Filippova GN, Henikoff S, et al. Trans- and cis-acting effects of Firre on epigenetic features of the inactive X chromosome. Nat Commun. 2020;11:6053.

14. Clarkson CT, Deeks EA, Samarista R, Mamayusupova H, Zhurkin VB, Teif VB. CTCF-dependent chromatin boundaries formed by asymmetric nucleosome arrays with decreased linker length. Nucleic Acids Res. 2019;47:11181-96.

15. Beishline K, Vladimirova O, Tutton S, Wang Z, Deng Z, Lieberman PM. CTCF driven TERRA transcription facilitates completion of telomere DNA replication. Nat Commun. 2017;8:2114.

16. Ong CT, Corces VG. CTCF: an architectural protein bridging genome topology and function. Nat Rev Genet. 2014;15:234-46.

17. Heath H, Ribeiro de Almeida C, Sleutels F, Dingjan G, van de Nobelen S, Jonkers I, et al. CTCF regulates cell cycle progression of alphabeta T cells in the thymus. EMBO J. 2008;27:2839-50.

18. Farrar D, Rai S, Chernukhin I, Jagodic M, Ito Y, Yammine S, et al. Mutational analysis of the poly(ADP-ribosyl)ation sites of the transcription factor CTCF provides an insight into the mechanism of its regulation by poly(ADP-ribosyl)ation. Mol Cell Biol. 2010;30:1199-216.

19. Tang Z, Luo OJ, Li X, Zheng M, Zhu JJ, Szalaj P, et al. CTCF-mediated human 3D genome architecture reveals chromatin topology for transcription. Cell. 2015;163:1611-27.

20. Katainen R, Dave K, Pitkänen E, Palin K, Kivioja T, Välimäki N, et al. CTCF/cohesin-binding sites are frequently mutated in cancer. Nat Genet. 2015;47:818-21.

21. Chen $\mathrm{X}, \mathrm{Ke} \mathrm{Y}, \mathrm{Wu} \mathrm{K}$, Zhao H, Sun Y, Gao L, et al. Key role for CTCF in establishing chromatin structure in human embryos. Nature. 2019;576:306-10.

22. Cao GW. Cancer Evo-Dev, a novel hypothesis derived from studies on hepatitis B virus-induced carcinogenesis. Hepatoma Res. 2017;3:241-9.

23. Atlasi Y, Stunnenberg HG. The interplay of epigenetic marks during stem cell differentiation and development. Nat Rev Genet. 2017;18:643-58.

24. Fullwood MJ, Liu MH, Pan YF, Liu J, Xu H, Mohamed YB, et al. An oestrogen-receptor-alpha-bound human chromatin interactome. Nature. 2009;462:58-64.

25. de Wit E, Vos ES, Holwerda SJ, Valdes-Quezada C, Verstegen MJ, Teunissen H, et al. CTCF binding polarity determines chromatin looping. Mol Cell. 2015;60:676-84.

26. Hnisz D, Day DS, Young RA. Insulated neighborhoods: structural and functional units of mammalian gene control. Cell. 2016;167:1188-200.

27. Ribeiro de Almeida C, Stadhouders R, de Bruijn MJ, Bergen IM, Thongjuea S, Lenhard B, et al. The DNA-binding protein CTCF limits proximal $V \kappa$ recombination and restricts $\kappa$ enhancer interactions to the immunoglobulin $\kappa$ light chain locus. Immunity. 2011;35:501-13.

28. Wan LB, Pan H, Hannenhalli S, Cheng Y, Ma J, Fedoriw A, et al. Maternal depletion of CTCF reveals multiple functions during oocyte and preimplantation embryo development. Development. 2008;135:2729-38.

29. Dowen JM, Fan ZP, Hnisz D, Ren G, Abraham BJ, Zhang LN, et al. Control of cell identity genes occurs in insulated neighborhoods in mammalian chromosomes. Cell. 2014;159:374-87.

30. Dixon JR, Jung I, Selvaraj S, Shen Y, Antosiewicz-Bourget JE, Lee AY, et al. Chromatin architecture reorganization during stem cell differentiation. Nature. 2015;518:331-6. 
31. Lieberman-Aiden E, van Berkum NL, Williams L, Imakaev M, Ragoczy T, Telling A, et al. Comprehensive mapping of long-range interactions reveals folding principles of the human genome. Science. 2009;326:289-93.

32. Nora EP, Goloborodko A, Valton AL, Gibcus JH, Uebersohn A, Abdennur N, et al. Targeted degradation of CTCF decouples local insulation of chromosome domains from genomic compartmentalization. Cell. 2017;169:930-44.e22.

33. Fiorentino FP, Giordano A. The tumor suppressor role of CTCF. J Cell Physiol. 2012;227:479-92.

34. Rasko JE, Klenova EM, Leon J, Filippova GN, Loukinov DI, Vatolin S, et al. Cell growth inhibition by the multifunctional multivalent zinc-finger factor CTCF. Cancer Res. 2001;61:6002-7.

35. Tiffen JC, Bailey CG, Marshall AD, Metierre C, Feng Y, Wang Q, et al. The cancer-testis antigen BORIS phenocopies the tumor suppressor CTCF in normal and neoplastic cells. Int J Cancer. 2013;133:1603-13.

36. Lang F, Li X, Zheng W, Li Z, Lu D, Chen G, et al. CTCF prevents genomic instability by promoting homologous recombination-directed DNA double-strand break repair. Proc Natl Acad Sci U S A. 2017;114:10912-7.

37. Demeyer A, Benhelli-Mokrani H, Chenais B, Weigel P, Fleury F. Inhibiting homologous recombination by targeting RAD51 protein. Biochim Biophys Acta Rev Cancer. 2021;1876:188597.

38. Voutsadakis IA. Molecular lesions of insulator CTCF and its paralogue CTCFL (BORIS) in cancer: an analysis from published genomic studies. High Throughput. 2018;7:30.

39. Zhang Y, Song Y, Li C, Ren J, Fang M, Fang J, et al. Brother of regulator of imprinted sites inhibits cisplatin-induced DNA damage in non-small cell lung cancer. Oncol Lett. 2020;20:251.

40. Kemp CJ, Moore JM, Moser R, Bernard B, Teater M, Smith LE, et al. CTCF haploinsufficiency destabilizes DNA methylation and predisposes to cancer. Cell Rep. 2014;7:1020-9.

41. Lai AY, Fatemi M, Dhasarathy A, Malone C, Sobol SE, Geigerman C, et al. DNA methylation prevents CTCF-mediated silencing of the oncogene BCL6 in B cell lymphomas. J Exp Med. 2010;207:1939-50.

42. Luo H, Yu Q, Liu Y, Tang M, Liang M, Zhang D, et al. LATS kinase-mediated CTCF phosphorylation and selective loss of genomic binding. Sci Adv. 2020;6:eaaw4651.

43. Nanavaty V, Abrash EW, Hong C, Park S, Fink EE, Li Z, et al. DNA methylation regulates alternative polyadenylation via CTCF and the cohesin complex. Mol Cell. 2020;78:752-64.e6.

44. Chang JW, Hsu HS, Ni HJ, Chuang CT, Hsiung CH, Huang TH, et al. Distinct epigenetic domains separated by a CTCF bound insulator between the tandem genes, BLU and RASSF1A. PloS One. 2010;5:e12847.

45. Kim TG, Kim S, Jung S, Kim M, Yang B, Lee MG, et al. CCCTC-binding factor is essential to the maintenance and quiescence of hematopoietic stem cells in mice. Exp Mol Med. 2017;49:e371.

46. Marshall AD, Bailey CG, Champ K, Vellozzi M, O’Young P, Metierre C, et al. CTCF genetic alterations in endometrial carcinoma are pro-tumorigenic. Oncogene. 2017;36:4100-10.

47. Lawrence MS, Stojanov P, Mermel CH, Robinson JT, Garraway LA, Golub TR, et al. Discovery and saturation analysis of cancer genes across 21 tumour types. Nature. 2014;505:495-501.

48. Aitken SJ, Ibarra-Soria X, Kentepozidou E, Flicek P, Feig C, Marioni JC, et al. CTCF maintains regulatory homeostasis of cancer pathways. Genome Biol. 2018;19:106.

49. Damaschke NA, Gawdzik J, Avilla M, Yang B, Svaren J, Roopra A, et al. CTCF loss mediates unique DNA hypermethylation landscapes in human cancers. Clin Epigenetics. 2020;12:80.

50. Hoffman B, Amanullah A, Shafarenko M, Liebermann DA. The proto-oncogene c-myc in hematopoietic development and leukemogenesis. Oncogene. 2002;21:3414-21.

51. van Riggelen J, Yetil A, Felsher DW. MYC as a regulator of ribosome biogenesis and protein synthesis. Nat Rev Cancer. 2010;10:301-9.

52. Dang CV, O'Donnell KA, Zeller KI, Nguyen T, Osthus RC, Li F. The c-Myc target gene network. Semin Cancer Biol. 2006;16:253-64. 
53. Jayapal SR, Lee KL, Ji P, Kaldis P, Lim B, Lodish HF. Down-regulation of Myc is essential for terminal erythroid maturation. J Biol Chem. 2010;285:40252-65.

54. Muller M. Cellular senescence: molecular mechanisms, in vivo significance, and redox considerations. Antioxid Redox Signal. 2009;11:59-98.

55. Masutomi K, Yu EY, Khurts S, Ben-Porath I, Currier JL, Metz GB, et al. Telomerase maintains telomere structure in normal human cells. Cell. 2003;114:241-53.

56. Cukusić A, Skrobot Vidacek N, Sopta M, Rubelj I. Telomerase regulation at the crossroads of cell fate. Cytogenet Genome Res. 2008;122:263-72.

57. Renaud S, Loukinov D, Bosman FT, Lobanenkov V, Benhattar J. CTCF binds the proximal exonic region of hTERT and inhibits its transcription. Nucleic Acids Res. 2005;33:6850-60.

58. Renaud S, Loukinov D, Abdullaev Z, Guilleret I, Bosman FT, Lobanenkov V, et al. Dual role of DNA methylation inside and outside of CTCF-binding regions in the transcriptional regulation of the telomerase hTERT gene. Nucleic Acids Res. 2007;35:1245-56.

59. Choi JH, Min NY, Park J, Kim JH, Park SH, Ko YJ, et al. TSA-induced DNMT1 down-regulation represses hTERT expression via recruiting CTCF into demethylated core promoter region of hTERT in HCT116. Biochem Biophys Res Commun. 2010;391:449-54.

60. Walter DM, Yates TJ, Ruiz-Torres M, Kim-Kiselak C, Gudiel AA, Deshpande C, et al. RB constrains lineage fidelity and multiple stages of tumour progression and metastasis. Nature. 2019;569:423-7.

61. De La Rosa-Velázquez IA, Rincón-Arano H, Benítez-Bribiesca L, Recillas-Targa F. Epigenetic regulation of the human retinoblastoma tumor suppressor gene promoter by CTCF. Cancer Res. 2007;67:2577-85.

62. Hollstein M, Hainaut P. Massively regulated genes: the example of TP53. J Pathol. 2010;220:164-73.

63. Rao SS, Huntley MH, Durand NC, Stamenova EK, Bochkov ID, Robinson JT, et al. A 3D map of the human genome at kilobase resolution reveals principles of chromatin looping. Cell. 2014;159:1665-80.

64. Nora E, Caccianini L, Fudenberg G, So K, Kameswaran V, Nagle A, et al. Molecular basis of CTCF binding polarity in genome folding. Nat Commun. 2020;11:5612.

65. Li Y, Haarhuis J, Sedeño Cacciatore Á, Oldenkamp R, van Ruiten M, Willems L, et al. The structural basis for cohesin-CTCF-anchored loops. Nature. 2020;578:472-6.

66. Merkenschlager M, Nora EP. CTCF and cohesin in genome folding and transcriptional gene regulation. Annu Rev Genomics Hum Genet. 2016;17:17-43.

67. Canela A, Maman Y, Jung S, Wong N, Callen E, Day A, et al. Genome organization drives chromosome fragility. Cell. 2017;170:507-21.e18.

68. Kon A, Shih LY, Minamino M, Sanada M, Shiraishi Y, Nagata Y, et al. Recurrent mutations in multiple components of the cohesin complex in myeloid neoplasms. Nat Genet. 2013;45:1232-7.

69. Dorsett $D$, Ström L. The ancient and evolving roles of cohesin in gene expression and DNA repair. Curr Biol. 2012;22:R240-50.

70. Yun J, Song SH, Kang JY, Park J, Kim HP, Han SW, et al. Reduced cohesin destabilizes high-level gene amplification by disrupting pre-replication complex bindings in human cancers with chromosomal instability. Nucleic Acids Res. 2016;44:558-72.

71. Surdez D, Zaidi S, Grossetête S, Laud-Duval K, Ferre AS, Mous L, et al. STAG2 mutations alter CTCF-anchored loop extrusion, reduce cis-regulatory interactions and EWSR1-FLI1 activity in Ewing sarcoma. Cancer cell. 2021;39:810-26.e9.

72. Yun J, Song SH, Kim HP, Han SW, Yi EC, Kim TY. Dynamic cohesin-mediated chromatin architecture controls epithelial-mesenchymal plasticity in cancer. EMBO Rep. 2016;17:1343-59.

73. Loukinov DI, Pugacheva E, Vatolin S, Pack SD, Moon H, Chernukhin I, et al. BORIS, a novel male germ-line-specific protein associated with epigenetic reprogramming events, shares the same 
11-zinc-finger domain with CTCF, the insulator protein involved in reading imprinting marks in the soma. Proc Natl Acad Sci U S A. 2002;99:6806-11.

74. Martin-Kleiner I. BORIS in human cancers-a review. Eur J Cancer. 2012;48:929-35.

75. Soltanian S, Dehghani H. BORIS: a key regulator of cancer stemness. Cancer Cell Int. 2018;18:154.

76. Nishana M, Ha C, Rodriguez-Hernaez J, Ranjbaran A, Chio E, Nora EP, et al. Defining the relative and combined contribution of CTCF and CTCFL to genomic regulation. Genome Biol. 2020;21:108.

77. Rivero-Hinojosa S, Pugacheva EM, Kang S, Méndez-Catalá CF, Kovalchuk AL, Strunnikov AV, et al. The combined action of CTCF and its testis-specific paralog BORIS is essential for spermatogenesis. Nat Commun. 2021;12:3846.

78. Pugacheva EM, Rivero-Hinojosa S, Espinoza CA, Méndez-Catalá CF, Kang S, Suzuki T, et al. Comparative analyses of CTCF and BORIS occupancies uncover two distinct classes of CTCF binding genomic regions. Genome Biol. 2015;16:161.

79. Höflmayer D, Steinhoff A, Hube-Magg C, Kluth M, Simon R, Burandt E, et al. Expression of CCCTC-binding factor (CTCF) is linked to poor prognosis in prostate cancer. Mol Oncol. 2020;14:129-38.

80. Zhang B, Zhang Y, Zou X, Chan AW, Zhang R, Lee TK, et al. The CCCTC-binding factor (CTCF)-forkhead box protein $\mathrm{M} 1$ axis regulates tumour growth and metastasis in hepatocellular carcinoma. J Pathol. 2017;243:418-30.

81. Amabile A, Migliara A, Capasso P, Biffi M, Cittaro D, Naldini L, et al. Inheritable silencing of endogenous genes by hit-and-run targeted epigenetic editing. Cell. 2016;167:219-32.e14.

82. Liu XS, Wu H, Ji X, Stelzer Y, Wu X, Czauderna S, et al. Editing DNA methylation in the mammalian genome. Cell. 2016;167:233-47.e17. 